\title{
Local Point Configurations of Discrete Combinatorial Surfaces
}

\author{
Yukiko Kenmochi ${ }^{1}$ and Yusuke Nomura ${ }^{2}$ \\ 1 UMR 8049 - IGM, CNRS/University of Marne-la-Vallée/ESIEE, France \\ 2 Department of Information Technology, Okayama University, Japan \\ y.kenmochi@esiee.fr
}

\begin{abstract}
Representing discrete objects by polyhedral complexes, we study topological properties of boundary points and surface points. We then obtain the local point configurations of discrete surfaces which are also considered to be boundaries of discrete objects.
\end{abstract}

\section{Introduction}

Surface structures are often used in the three-dimensional image analysis, for example, an active balloon which is a deformable surface for the image segmentation [2. In many cases, a continuous surface such as a spline surface is applied to a deformable surface and after its deformation it is rediscretized to be a discrete object in a digital image. Obviously, this is not efficient if the input and output are both discrete. To construct completely discrete methods similarly to reference 4, we study discrete surfaces and their local point configurations such as those in the $3 \times 3 \times 3$ region in this paper as our first step.

In our previous work [10, we presented a boundary tracking algorithm which provides a triangulation of a set of boundary points given by

$$
B r_{m}(\mathbf{V})=\left\{\boldsymbol{x} \in \mathbf{V}: \mathbf{N}_{m}(\boldsymbol{x}) \cap \overline{\mathbf{V}} \neq \emptyset\right\}
$$

where $\mathbf{V}$ is the input, i.e., a discrete object in a three-dimensional image and $\overline{\mathbf{V}}$ is the complement. $\mathbf{N}_{m}(\boldsymbol{x})$ is the $m$-neighborhood of a point $\boldsymbol{x}=(i, j, k)$ in a threedimensional discrete space $\mathcal{Z}^{3}$, consisting of lattice points whose coordinates are all integers in $\mathcal{R}^{3}$, defined by

$$
\mathbf{N}_{m}(\boldsymbol{x})=\left\{(p, q, r) \in \mathcal{Z}^{3}:(i-p)^{2}+(j-q)^{2}+(k-r)^{2} \leq t\right\}
$$

where $t=1,3$ for $m=6,26$ respectively. Because we apply discrete polyhedral complexes [10] based on combinatorial topology [1,12,13] to object representation, we can obtain topologies for boundary points. With a help of the topologies, we found that our boundary points include not only surface points, i.e., points on combinatorial 2-manifold, but also non-surface points, i.e., singular points, as shown in Fig. 1. In this paper, we use local topological notions similarly to our work 8 to discriminate boundary points and also surface points. Such notions enable us to present an algorithm to count the local point configurations of discrete surfaces for the 6- and 26-neighborhood systems. 


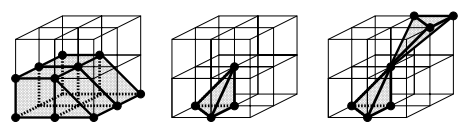

Fig. 1. Examples of point configurations in the $3 \times 3 \times 3$ region so that the central point is considered to be a boundary point 10; ; surface point (left), a surface point but not a simplicity surface point [3] (center), and a non-surface point, i.e., a singular point (right)

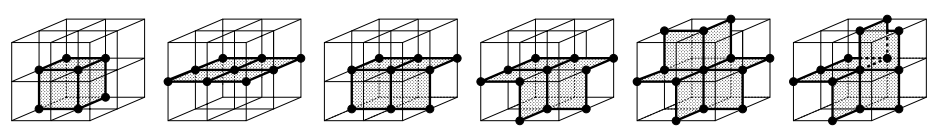

Fig. 2. The 6 local configurations of $3 \times 3 \times 3$ points for discrete combinatorial surfaces in the 6-neighborhood system

For the 6-neighborhood system, the definition of discrete combinatorial are given by Françon in [5] and he showed that there are 6 local configurations of discrete surfaces for the 6-neighborhood system as illustrated in Fig. 2. Note that the similar results are obtained by using different approaches, for example, in [7. The discrete deformation model based on such discrete surface configurations for 6-neighborhood system is also presented in [4.

In [5], however, the 26-neighborhood is not practically treated so that we do not see how to generate discrete combinatorial surfaces for the 26-neighborhood system, Morgenthaler et al. defined discrete surfaces by using the point connectivity based on the Jordan surface theorem; any Jordan surface divides the space into two [11]. In [3, Couprie et al. pointed out that, for the 26-neighborhood system, Morgenthaler's discrete surfaces have only 13 local point configurations while their discrete surfaces, called simplicity surfaces, have 736 configurations. However, we see that even simplicity surfaces do not give enough configurations if we would like to treat our boundary points. For example, we obtain a boundary point as illustrated in Fig. 1 (center) which is not considered to be a simplicity surface.

In this paper, we show that the surface configurations which appear on our boundaries for the 6 -neighborhood system are the same 6 configurations as illustrated in Fig. 2 and derive new results of surface configurations for the 26neighborhood system. We also discuss the utilities of such study on local point configurations of discrete surfaces for three-dimensional shape analysis.

\section{Discrete Polyhedral Complexes}

\subsection{Definitions}

In combinatorial topology, any object in a 3 -dimensional Euclidean space $\mathcal{R}^{3}$ is represented by a set of simplexes [1, 12] or more generally convex polyhedra [1, 13. We construct convex polyhedra whose vertices are lattice points in $\mathcal{Z}^{3}$. Such 
Table 1. All possible discrete convex polyhedra for the 6- and 26-neighborhood systems

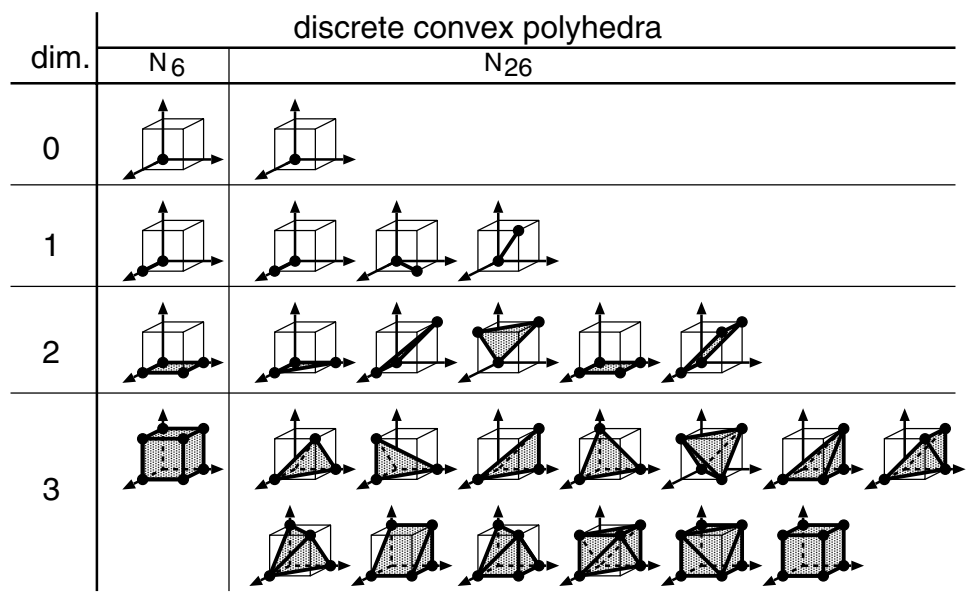

convex polyhedra are called discrete convex polyhedra, and a discrete polyhedral complex is constructed as a set of discrete convex polyhedra combined together without contradiction [10].

Let us consider a set of eight lattice points which are the vertices of a unit cube. Setting a value of each point to be 1 or 0 and calling such a point 1or 0 -point, we obtain $2^{8}$ different 1-point configurations in a unit cube. Up to symmetries and rotations, the number of different configurations becomes 23 . For each configuration which has at least one 1-point in a unit cube, we make a convex hull of 1-points and call it a discrete convex polyhedron.

Definition 1. If a convex hull of 1-points in a unit cube has $n$ dimensions where $n=0,1,2,3$ and its adjacent vertices are $m$-neighboring for $m=6,26$, such a convex hull is called an n-dimensional discrete convex polyhedron for the $m$ neighborhood system.

Table 1 shows $n$-dimensional discrete convex polyhedra, $n=0,1,2,3$, for the 6- and 26-neighborhood systems. Hereafter, we abbreviate $n$-dimensional discrete convex polyhedra to $n$-polyhedra. The face of an $r$-polyhedron $\sigma$ is defined to be the set of all $s$-polyhedra which are included in the boundary of $\sigma$ where $s<r$, denoted by $f a c e(\sigma)$. For example, we see in Table 1 that a 3-polyhedron for the 6-neighborhood system includes eight 0-polyhedra, twelve 1-polyhedra, and six 2-polyhedra. By using faces, we define discrete polyhedral complexes.

Definition 2. A discrete polyhedral complex $\mathbf{K}$ is the set of discrete convex polyhedra satisfying the following conditions:

1. $\emptyset \in \mathbf{K}$;

2. if $\sigma \in \mathbf{K}, \operatorname{face}(\sigma) \subset \mathbf{K}$;

3. if $\sigma, \tau \in \mathbf{K}, \sigma \cap \tau=\cup_{a \in \operatorname{face}(\sigma) \cap \operatorname{face}(\tau)} a$. 

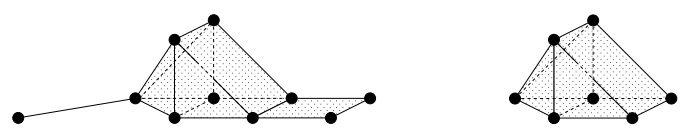

Fig. 3. Non-pure (left) and pure (right) 3-complexes

The dimension of $\mathbf{K}$ is defined as the maximum dimension of discrete convex polyhedra belonging to $\mathbf{K}$. Hereafter, we abbreviate an $n$-dimensional discrete polyhedral complex to an $n$-dimensional discrete complex or simply an $n$-complex. We will present several properties of discrete complexes.

Definition 3. Let $\mathbf{K}$ be an n-complex. If we have at least one $n$-polyhedron $\sigma \in \mathbf{K}$ for every s-polyhedron $\tau \in \mathbf{K}$ where $s<n$ satisfying $\tau \in$ face $(\sigma)$, then $\mathbf{K}$ is said to be pure.

Figure 3 shows examples of pure and non-pure discrete complexes. The 3complex in Fig. 3 (left) is not pure because it includes 0-, 1- and 2-polyhedra which do not belong to any 3-polyhedra. If we remove these 0-, 1- and 2polyhedra from Fig. 3 (left), we obtain a pure 3-complex in Fig. 3 (right).

Definition 4. Let $\mathbf{K}$ be a discrete complex. The combinatorial closure of a subset $\mathbf{K}_{0} \subset \mathbf{K}$ is defined as

$$
C l\left(\mathbf{K}_{0}\right)=\mathbf{K}_{0} \cup\left(\underset{a \in \mathbf{K}_{0}}{\cup} f a c e(a)\right) .
$$

Definition 5. Let $\mathbf{K}$ be a discrete complex, and $\sigma, \tau$ be arbitrary elements in $\mathbf{K}$. We say that $\mathbf{K}$ is connected, if we have a path $\sigma=a_{1}, a_{2}, \ldots, \tau=a_{n}$ which satisfies the following conditions:

1. $a_{i} \in \mathbf{K}$ for every $i=1,2, \ldots, n$;

2. $C l\left(\left\{a_{i}\right\}\right) \cap C l\left(\left\{a_{i+1}\right\}\right) \neq \emptyset$ for every $i=1,2, \ldots, n-1$.

\subsection{Discrete Complex Construction from a Lattice Point Set}

The goal of this paper is to count the number of local configurations of boundary points which form discrete surfaces, in a $3 \times 3 \times 3$ region $\mathbf{N}_{26}(\boldsymbol{x})$ of $\mathcal{Z}^{3}$ by using topological properties of discrete complexes presented in the next section. Before obtaining topological properties, thus, we need to construct a discrete complex $\mathbf{K}_{m}$ for $m=6,26$ from a subset $\mathbf{V} \subseteq \mathbf{N}_{26}(\boldsymbol{x})$ where all points in $\mathbf{V}$ (resp. the complement $\overline{\mathbf{V}}$ ) are 1-points (resp. 0-points). Given a $\mathbf{V} \subseteq \mathbf{N}_{26}(\boldsymbol{x})$, we briefly explain how to construct a discrete complex $\mathbf{K}_{m}$ for each $m=6,26$.

Let us first consider the case of $m=26$. From Definition 1, dividing a $3 \times 3 \times 3$ region $\mathbf{N}_{26}(\boldsymbol{x})$ into eight $2 \times 2 \times 2$ unit cubic regions, we obtain a discrete convex polyhedron by constructing a convex hull of 1-points in each unit cubic region. From Definition 2] collecting all discrete convex polyhedra for eight unit cubic regions, we finally obtain a discrete complex $\mathbf{K}_{26}$ such as a set of all discrete convex polyhedra and their faces.

For the case of $m=6$, we only consider convex hulls whose adjacent vertices are all 6-neighboring, and obtain a discrete complex $\mathbf{K}_{6}$ similarly to $\mathbf{K}_{26}$. The details and the precise algorithm are presented in reference [10]. 


\section{Point Classification by Combinatorial Topology}

In $\mathcal{Z}^{3}$, discrete complexes whose dimensions are from zero to three can exist. In this section, we first present topological properties of discrete complexes for each dimension from one to three by using the notions of star and link 13 similarly to the previous work [8]. By the topological properties, we classify all points in the skeleton of $\mathbf{K}$, denoted by $S k(\mathbf{K})$, which is the set of vertices of all discrete convex polyhedra in $\mathbf{K}$, and find out the topological type of boundary points which are considered to be on discrete surfaces.

\subsection{Star and Link}

The star and the link are defined for each vertex in a discrete complex as follows.

Definition 6. For a discrete complex $\mathbf{K}$, the star of a point $\boldsymbol{x} \in S k(\mathbf{K})$ is defined so that

$$
\operatorname{star}(\boldsymbol{x})=\{\sigma \in \mathbf{K}: \boldsymbol{x} \in \sigma\} .
$$

Definition 7. For a discrete complex $\mathbf{K}$, the link of a point $\boldsymbol{x} \in S k(\mathbf{K})$ is defined so that

$$
\operatorname{link}(\boldsymbol{x})=C l(\operatorname{star}(\boldsymbol{x})) \backslash \operatorname{star}(\boldsymbol{x}) .
$$

The star and link with respect to $\mathbf{K}$ are denoted by $\operatorname{star}(\boldsymbol{x}: \mathbf{K})$ and $\operatorname{link}(\boldsymbol{x}$ : $\mathbf{K})$, respectively, when we emphasize $\mathbf{K}$. Similarly to discrete complexes, we define the dimension of $\operatorname{star}(\boldsymbol{x}: \mathbf{K})$ as the maximum dimension of discrete convex polyhedra belonging to $\operatorname{star}(\boldsymbol{x}: \mathbf{K})$ and denoted by $\operatorname{dim}(\operatorname{star}(\boldsymbol{x}: \mathbf{K}))$. Note that $\operatorname{star}(\boldsymbol{x}: \mathbf{K})$ is not always a discrete complex because it may not satisfy the second condition in Definition 2 On the other hand, $\operatorname{link}(\boldsymbol{x}: \mathbf{K})$ always becomes a discrete complex and even pure if $\mathbf{K}$ is pure.

\subsection{Topological Properties in One Dimension}

If a 1-complex $\mathbf{K}$ is pure, stars of points in $S k(\mathbf{K})$ are classified into the following three types: linear stars, semi-linear stars, and neither of them. Let $|\mathbf{A}|$ be the number of elements in a set $\mathbf{A}$.

Definition 8. Let $\mathbf{K}$ be a pure 1-complex. If $|\operatorname{link}(\boldsymbol{x})|=2$, star $(\boldsymbol{x})$ is called linear.

Definition 9. Let $\mathbf{K}$ be a pure 1-complex. If $|\operatorname{link}(\boldsymbol{x})|=1$, star $(\boldsymbol{x})$ is called semi-linear.

Figure 4 illustrates points whose stars are linear, semi-linear and neither of them. We see that a point is an endpoint of a curve if its star is semi-linear, and an intermediate point of a curve if its star is linear. If the star of a point is neither linear nor semi-linear, it is an intersection of a curve. By using the above definitions, we define discrete curves in $\mathcal{Z}^{3}$. 


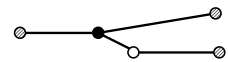

Fig. 4. Examples of points whose stars are linear, semi-linear and neither of them, illustrated as white, grey and black points, respectively

Definition 10. Let $\mathbf{K}$ be a connected and pure 1-complex. If the star of every point in $S k(\mathbf{K})$ is either linear or semi-linear and at least one point whose star is semi-linear exists in $S k(\mathbf{K}), \mathbf{K}$ is called a discrete curve with endpoints.

Definition 11. Let $\mathbf{K}$ be a connected and pure 1-complex. If the star of every point in $S k(\mathbf{K})$ is linear, $\mathbf{K}$ is called a discrete closed curve.

\subsection{Topological Properties in Two Dimensions}

If a 2-complex $\mathbf{K}$ is pure, stars of points in $S k(\mathbf{K})$ are classified into the following three types: cyclic stars, semi-cyclic stars, and neither of them.

Definition 12. Let $\mathbf{K}$ be a pure 2-complex. If link $(\boldsymbol{x})$ is a discrete closed curve, $\operatorname{star}(\boldsymbol{x})$ is cyclic.

Definition 13. Let $\mathbf{K}$ be a pure 2-complex. If $\operatorname{link}(\boldsymbol{x})$ is a discrete curve with endpoints, star $(\boldsymbol{x})$ is semi-cyclic.

Figure 5 illustrates points of stars which are cyclic, semi-cyclic and neither of them. We see that a point is an edge point of a surface if its star is semi-cyclic, and an interior point of a surface if its star is cyclic. If the star of a point is neither cyclic nor semi-cyclic, the point is at an intersection of surfaces. What we call local surface structures or configurations in this paper are, thus, cyclic stars. By using these properties, we define discrete surfaces in $\mathcal{Z}^{3}$.

Definition 14. Let $\mathbf{K}$ be a connected and pure 2-complex. If the star of every point in $S k(\mathbf{K})$ is either cyclic or semi-cyclic, and at least one point whose star is semi-cyclic exists in $S k(\mathbf{K}), \mathbf{K}$ is called a discrete surface with edges.

Definition 15. Let $\mathbf{K}$ be a connected and pure 2-complex. If the star of every point in $S k(\mathbf{K})$ is cyclic, $\mathbf{K}$ is called a discrete closed surface.

We see that discrete closed surfaces are considered to be 2-dimensional discrete combinatorial manifolds as presented in reference [5].

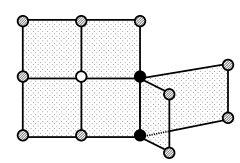

Fig. 5. Examples of points whose stars are cyclic, semi-cyclic and neither of them, illustrated as white, grey and black points, respectively 


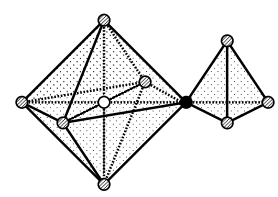

Fig. 6. Examples of points whose stars are spherical, semi-spherical and neither of them, illustrated as white, grey and black points, respectively

\subsection{Topological Properties in Three Dimensions}

If a 3-complex $\mathbf{K}$ is pure, stars of points in $S k(\mathbf{K})$ are classified into the following three types: spherical stars, semi-spherical stars, and neither of them.

Definition 16. Let $\mathbf{K}$ be a pure 3-complex. If $\operatorname{link}(\boldsymbol{x})$ is a discrete closed surface, $\operatorname{star}(\boldsymbol{x})$ is spherical.

We define semi-spherical stars by using the notion of combinatorial boundary given by Definition 4 .

Definition 17. Let $\mathbf{K}$ be a pure $n$-complex and $\mathbf{H}$ be the set of all $(n-1)$ polyhedra in $\mathbf{K}$ each of which is a face of exactly one n-polyhedron in $\mathbf{K}$. The combinatorial boundary of $\mathbf{K}$ is then defined as a pure $(n-1)$-complex such that

$$
\partial \mathbf{K}=C l(\mathbf{H}) .
$$

We now see that the endpoints of a discrete curve and the edges of a discrete surface in Definitions 10 and 14 are their combinatorial boundaries.

Definition 18. Let $\mathbf{K}$ be a connected and pure 3-complex. If link $(\boldsymbol{x})$ is a discrete surface with edges, and the edges, i.e., the combinatorial boundary $\partial(\operatorname{link}(\boldsymbol{x}))$ is a discrete closed curve, then star $(\boldsymbol{x})$ is semi-spherical.

Figure 6 illustrates points whose stars are spherical, semi-spherical and neither of them. It shows that points whose stars are spherical and non-spherical considered to be interior and boundary points of a 3-complex, respectively. If the stars of boundary points are not semi-spherical, such points are considered to be singular points, i.e., intersection points of the boundaries.

In the previous work [8], $\operatorname{star}(\boldsymbol{x})$ is simply defined to be semi-spherical if $\operatorname{link}(\boldsymbol{x})$ is a discrete surface with edges. However, we found a counter-example such as a white central point in Fig. 7 (left); its link is a discrete surface with two combinatorial boundaries, and its star should not be regarded as a semispherical star because it is not topologically equivalent to a semi-sphere. We therefore modify our definition of semi-spherical stars.

Such modification enables us to prove the following important proposition.

Proposition 1. Let $\mathbf{K}$ be a pure 3-complex. If star $(\boldsymbol{x}: \mathbf{K})$ is semi-spherical, then $\operatorname{star}(\boldsymbol{x}: \partial \mathbf{K})$ is cyclic. 

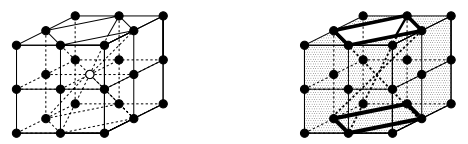

Fig. 7. An example of a point whose star is considered to be semi-spherical with our previous definition in [8], but not semi-spherical with that in this paper, illustrated as the white point in the left figure. Its link and its combinatorial boundary are illustrated as a half-tone region and two black bold closed curves in the right figure

Before proving this proposition, we first derive the next lemma.

Lemma 1. Let $\mathbf{K}$ be a pure 3-complex. If $\operatorname{star}(\boldsymbol{x}: \mathbf{K})$ is semi-spherical, then

$$
\partial(\operatorname{link}(\boldsymbol{x}: \mathbf{K}))=\operatorname{link}(\boldsymbol{x}: \partial \mathbf{K}) .
$$

(Proof) From Definitions 7 and 17 (or [18), we see that both $\partial(\operatorname{link}(\boldsymbol{x}: \mathbf{K}))$ and $\operatorname{link}(\boldsymbol{x}: \partial \mathbf{K})$ are pure 1-complexes. Thus, we need to prove simply for 1-polyhedra $\sigma$ that $\sigma \in \operatorname{link}(\boldsymbol{x}: \partial \mathbf{K})$ if and only if $\sigma \in \partial(\operatorname{link}(\boldsymbol{x}: \mathbf{K}))$.

Let $\sigma$ be each 1-polyhedron in $\partial(\operatorname{link}(\boldsymbol{x}: \mathbf{K}))$. Then $\sigma$ is a face of some 2-polyhedron $\tau$ in $\operatorname{star}(\boldsymbol{x}: \mathbf{K})$. In $\operatorname{star}(\boldsymbol{x}: \mathbf{K})$, we have two different types of 2-polyhedra $\tau$ such that $\tau \in \partial \mathbf{K}$ or $\tau \notin \partial \mathbf{K}$. Now, we show that $\tau \in \partial \mathbf{K}$ if $\sigma \in \partial(\operatorname{link}(\boldsymbol{x}: \mathbf{K}))$ by proving its contraposition. If $\tau \notin \partial \mathbf{K}, \tau$ becomes a shared face of two 3-polyhedra in $\operatorname{star}(\boldsymbol{x}: \mathbf{K})$, therefore $\sigma$ becomes a shared face of two 2-polyhedra in $\operatorname{link}(\boldsymbol{x}: \mathbf{K})$. This means that $\sigma \notin \partial(\operatorname{link}(\boldsymbol{x}: \mathbf{K}))$. Because $\tau \in \operatorname{star}(\boldsymbol{x}: \mathbf{K})$ and $\tau \in \partial \mathbf{K}, \tau \in \operatorname{star}(\boldsymbol{x}: \partial \mathbf{K})$. Therefore, if $\sigma \in \partial(\operatorname{link}(\boldsymbol{x}: \mathbf{K}))$, $\sigma \in \operatorname{link}(\boldsymbol{x}: \partial \mathbf{K})$.

Next, we show that $\sigma \in \partial(\operatorname{link}(\boldsymbol{x}: \mathbf{K}))$ if $\sigma \in \operatorname{link}(\boldsymbol{x}: \partial \mathbf{K})$ contrarily. For any 1-polyhedron $\sigma \in \operatorname{link}(\boldsymbol{x}: \partial \mathbf{K})$, we have a 2-polyhedron $\tau \in \partial \mathbf{K}$ such that $\sigma \in \operatorname{face}(\tau)$. Such $\tau$ is in $\operatorname{star}(\boldsymbol{x}: \mathbf{K})$, and it is not a shared face of two 3 -polyhedra in $\operatorname{star}(\boldsymbol{x}: \mathbf{K})$ but there is exactly one 3-polyhedron $v$ such that $\tau \in$ face $(v)$. Therefore, $\sigma$ is not a shared face of two 2-polyhedra in $\operatorname{link}(\boldsymbol{x}: \mathbf{K})$, and from Definition 17, $\sigma \in \partial(\operatorname{link}(\boldsymbol{x}: \mathbf{K}))$.

(Q.E.D.)

(Proof of Proposition 1) From Definition 18, if $\operatorname{star}(\boldsymbol{x}: \mathbf{K})$ is semi-spherical, $\partial(\operatorname{link}(\boldsymbol{x}: \mathbf{K}))$ is a discrete closed curve. We then obtain from Lemma 1 that $\operatorname{link}(\boldsymbol{x}: \partial \mathbf{K})$ is also a discrete closed curve. Thus, from Definition 12, $\operatorname{star}(\boldsymbol{x}$ : $\partial \mathbf{K})$ is cyclic.

(Q.E.D.)

\subsection{Topological Point Classification}

By using topological properties defined above, we classify points $\boldsymbol{x} \in S k(\mathbf{K})$ into the following twelve types. Note that each point in $S k(\mathbf{K})$ is classified into one of them.

Type 0: $\operatorname{dim}(\operatorname{star}(\boldsymbol{x}))=0$;

Type 1a: $\operatorname{dim}(\operatorname{star}(\boldsymbol{x}))=1$ and $\operatorname{star}(\boldsymbol{x})$ is linear;

Type 1b: $\operatorname{dim}(\operatorname{star}(\boldsymbol{x}))=1$ and $\operatorname{star}(\boldsymbol{x})$ is semi-linear; 
Type 1c: $\operatorname{dim}(\operatorname{star}(\boldsymbol{x}))=1$ and $\operatorname{star}(\boldsymbol{x})$ is neither linear nor semi-linear;

Type 2a: $\operatorname{dim}(\operatorname{star}(\boldsymbol{x}))=2, C l(\operatorname{star}(\boldsymbol{x}))$ is pure and $\operatorname{star}(\boldsymbol{x})$ is cyclic;

Type 2b: $\operatorname{dim}(\operatorname{star}(\boldsymbol{x}))=2, C l(\operatorname{star}(\boldsymbol{x}))$ is pure and $\operatorname{star}(\boldsymbol{x})$ is semi-cyclic;

Type 2c: $\operatorname{dim}(\operatorname{star}(\boldsymbol{x}))=2, C l(\operatorname{star}(\boldsymbol{x}))$ is pure and $\operatorname{star}(\boldsymbol{x})$ is neither cyclic nor semi-cyclic;

Type 2d: $\operatorname{dim}(\operatorname{star}(\boldsymbol{x}))=2$ and $C l(\operatorname{star}(\boldsymbol{x}))$ is not pure;

Type 3a: $\operatorname{dim}(\operatorname{star}(\boldsymbol{x}))=3, C l(\operatorname{star}(\boldsymbol{x}))$ is pure and $\operatorname{star}(\boldsymbol{x})$ is spherical;

Type 3b: $\operatorname{dim}(\operatorname{star}(\boldsymbol{x}))=3, C l(\operatorname{star}(\boldsymbol{x}))$ is pure and $\operatorname{star}(\boldsymbol{x})$ is semi-spherical;

Type 3c: $\operatorname{dim}(\operatorname{star}(\boldsymbol{x}))=3, C l(\operatorname{star}(\boldsymbol{x}))$ is pure and $\operatorname{star}(\boldsymbol{x})$ is neither spherical nor semi-spherical;

Type 3d: $\operatorname{dim}(\operatorname{star}(\boldsymbol{x}))=3$ and $C l(\operatorname{star}(\boldsymbol{x}))$ is not pure.

Our discrete surfaces are considered to appear at the 2-dimensional combinatorial boundaries of 3 -complexes, that is, $\partial \mathbf{K}$ where $\operatorname{dim}(\mathbf{K})=3$. From Proposition 1, we see that our points of interest whose local point configurations form discrete surfaces have type $3 \mathrm{~b}$.

\section{Local Point Configurations of Discrete Surfaces}

Let us consider a 1-point $\boldsymbol{x} \in \mathcal{Z}^{3}$ with a set $\mathbf{V}$ of its neighboring 1-points in $\mathbf{N}_{26}(\boldsymbol{x})$ and a discrete complex $\mathbf{K}_{m}$, for $m=6,26$, constructed from $\mathbf{V} \subseteq \mathbf{N}_{26}(\boldsymbol{x})$ as explained in the subsection 2.2. In this section, we present an algorithm for examining whether $\boldsymbol{x}$ is a boundary point considered to be on a discrete surface, i.e., $\operatorname{star}(\boldsymbol{x}: \partial \mathbf{K})$ is cyclic or $\operatorname{star}(\boldsymbol{x}: \mathbf{K})$ is semi-spherical, for a given set $\mathbf{V}$. It means that we check if the type of $\boldsymbol{x}$ is $3 \mathrm{~b}$. We apply it for all possible configurations of $\mathbf{V}$ to count the number of local point configurations which form discrete surfaces.

\subsection{Algorithm}

Setting the value of $\boldsymbol{x}$ to be 1, for any configuration of a 1-point set $\mathbf{V} \subseteq \mathbf{N}_{26}(\boldsymbol{x})$, we apply the following algorithm which returns 1 if $\boldsymbol{x}$ is type $3 \mathrm{~b}$.

\section{Algorithm 1}

Input: $A$ 1-point set $\mathbf{V}$ in a $3 \times 3 \times 3$ region of $\mathcal{Z}^{3}$.

Output: If the central point $\boldsymbol{x}$ has type $3 b$, return 1 , otherwise, return 0 . begin

1. construct $\mathbf{K}_{m}$ from $\mathbf{V}$ choosing $m=6$ or 26 ;

2. obtain $\operatorname{star}\left(\boldsymbol{x}: \mathbf{K}_{m}\right)$;

3. if $\operatorname{dim}\left(\operatorname{star}\left(\boldsymbol{x}: \mathbf{K}_{m}\right)\right) \neq 3$ then return 0 ;

4. if $C l\left(\operatorname{star}\left(\boldsymbol{x}: \mathbf{K}_{m}\right)\right)$ is not pure then return 0 ;

5. if $\operatorname{star}\left(\boldsymbol{x}: \mathbf{K}_{m}\right)$ is semi-spherical then return 1; else return 0 ; 

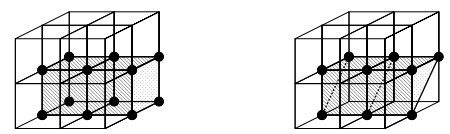

Fig. 8. Two different discrete complexes $\mathbf{K}_{26}$ (left) and $\mathbf{K}_{26}^{\prime}$ (right) around the central points $\boldsymbol{x}$ so that $\operatorname{star}\left(\boldsymbol{x}: \partial \mathbf{K}_{26}\right)=\operatorname{star}\left(\boldsymbol{x}: \partial \mathbf{K}_{26}^{\prime}\right)$

\subsection{Experiments}

By using Algorithm 1, we count the 1-point configurations of $\mathbf{V}$, namely $S k\left(\mathbf{K}_{m}\right)$, where the central point $\boldsymbol{x}$ is type $3 \mathrm{~b}$. They are called surface complicial configurations. Up to rotations and symmetries, the number of all 1-point configurations of $\mathbf{V}$ is reduced to $1,426,144$ from $2^{26}=67,108,864$. Among them, we obtain 14,031 and 290, 979 surface complicial configurations for the 6- and 26neighborhood systems, respectively. We also consider the point configurations of $S k\left(C l\left(\operatorname{star}\left(\boldsymbol{x}: \partial \mathbf{K}_{m}\right)\right)\right.$ where $\boldsymbol{x}$ is type $3 \mathrm{~b}$ to see only the surface structures around $\boldsymbol{x}$, that is $\operatorname{star}\left(\boldsymbol{x}: \partial \mathbf{K}_{m}\right)$. They are called surface star configurations. We then see that there are different discrete complexes $\mathbf{K}_{m}$ and $\mathbf{K}_{m}^{\prime}$ such that $\operatorname{star}\left(\boldsymbol{x}: \partial \mathbf{K}_{m}\right)=\operatorname{star}\left(\boldsymbol{x}: \partial \mathbf{K}_{m}^{\prime}\right)$, as illustrated in Fig. 8, and they have the same surface star configurations. We count surface star configurations and finally obtain 6 and 6,028 for $m=6,26$, respectively.

\section{Conclusions}

Given a subset $\mathbf{V} \subseteq \mathbf{N}_{26}(\boldsymbol{x})$, we presented a method for classifying the central point $\boldsymbol{x}$ into one of the twelve types by the topological property of its star after obtaining a complex $\mathbf{K}_{m}$. With our conclusion such that the type of points which are boundary points having local point configurations of discrete surfaces is $3 \mathrm{~b}$, we counted surface complicial configurations $S k\left(\mathbf{K}_{m}\right)$ and obtained 14,031 and 290,979 configurations for $m=6,26$ up to symmetries and rotations. We also obtained 6 and 6,028 surface star configurations, namely, $\operatorname{Sk}(C l(\operatorname{star}(\boldsymbol{x}$ : $\left.\left.\partial \mathbf{K}_{m}\right)\right)$ ), for $m=6,26$. The same result for $m=6$ is already presented in reference 5 and they are illustrated in Fig. 2. We see that a boundary point illustrated as the central point in Fig. 1 (center) has a surface star configuration. This explains why our discrete surfaces have more configurations than that of simplicity surfaces 3 .

References 6, 9] show that there are 5 and 32 different configurations of stars which appear in discrete combinatorial planes for $m=6,26$, respectively. Such planar stars for $m=6$ are shown as the five left configurations in Fig. 2 , We also illustrate the 32 configurations of planar stars for $m=26$ in Fig. 9. Note that oriented surfaces are considered in [6, 9] and the 8 and 34 configurations are obtained for $m=6,26$. While there is only one non-planar star for $m=6$, we see that, for $m=26$, most of the 6,028 , namely 5,994 surface stars are non-planar and they do not appear on discrete planes but appear on discrete non-planar surfaces. Figure 10 shows that, for example, every boundary point 


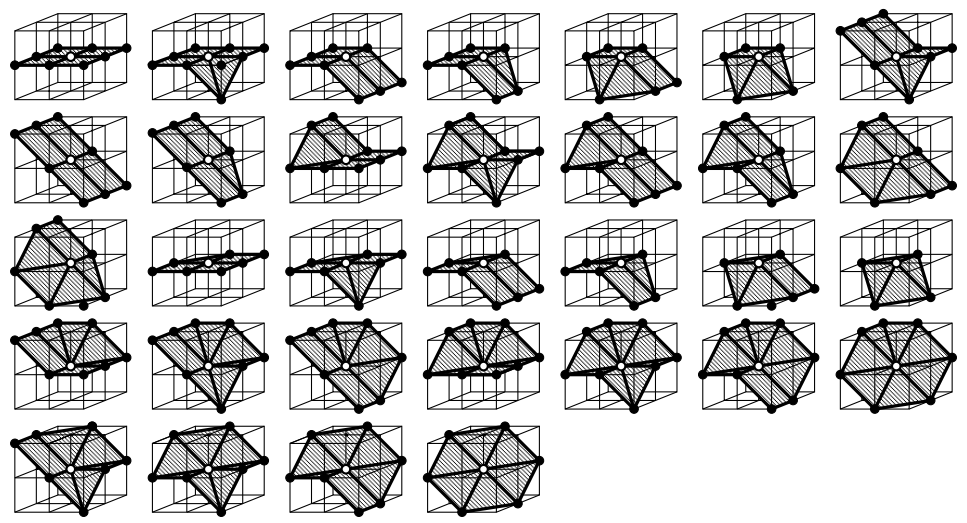

Fig. 9. The 32 local star configurations which appear in discrete combinatorial planes for the 26-neighborhood system
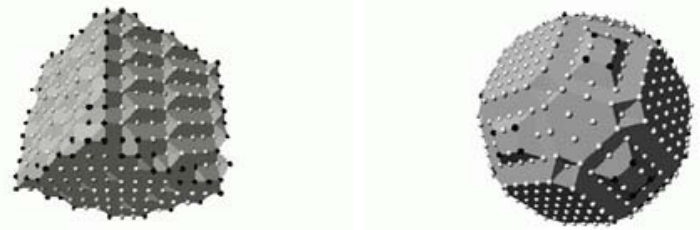

Fig. 10. Boundary points of three-dimensional digitized objects, such as a cube and a sphere are classified into two types in the 26-neighborhood system: they are illustrated as white and black points if the stars are planar and non-planar, respectively

appearing at the faces of a digitized cube has one of the 32 planar stars illustrated in Fig. 9. On the other hand, around the vertices and edges of a digitized cube, boundary points have non-planar stars. Figure 10 also shows that many boundary points on non-planar surfaces such as a sphere have planar stars rather than non-planar stars. From such experiments, we consider that the study on local configurations of boundary points in the 26-neighborhood system might be useful for shape analysis of three-dimensional images. We remark that the similar shape analysis may not be worth doing for the 6-neighborhood system because most of all boundary points of three-dimensional digitized objects have planar stars.

Acknowledgements. A part of this work was supported by Grant-in-Aid for Scientific Research of the Ministry of Education, Culture, Sports, Science and Technology of Japan under the contract of 15700152 and 16650040. 


\section{References}

1. P. S. Alexandrov, Combinatorial Topology, Vol. 1, Graylock Press, Rochester, New York, 1956.

2. L. D. Cohen, "On active contour models and balloons," Computer Vision, Graphics and Image Processing: Image Understanding, Vol. 53, No. 2, pp. 211-218, 1991.

3. M. Couprie, G. Bertrand, "Simplicity surface: a new definition of surfaces in $\mathbf{Z}^{3}$," In Vision Geometry VII, Proceedings of SPIE, Vol. 3454, pp. 40-51, 1998.

4. A. Esnard, J-O. Lachaud, A. Vialard, "Discrete deformable boundaries for 3D image segmentation," Technical Report No. 1270-02, LaBRI, University of Bordeaux $1,2002$.

5. J. Françon, "Discrete combinatorial surfaces," Graphical Models and Image Processing, Vol. 57, No. 1, pp. 20-26, 1995.

6. J. Françon, "Sur la topologie d'un plan arithmétique," Theoretical Computer Science, Vol. 156, pp. 159-176, 1996.

7. P. P. Jonker, S. Svensson, "The generation of $\mathrm{N}$ dimensional shape primitives," in LNCS 2886 : Discrete Geometry for Computer Imagery, Proceedings of 11th International Conference, DGCI2003, pp.420-433, Springer-Verlag, Heidelberg, 2003.

8. Y. Kenmochi, A. Imiya, A. Ichikawa, "Discrete combinatorial geometry," Pattern Recognition, Vol. 30, No. 10, pp. 1719-1728, 1997.

9. Y. Kenmochi, A. Imiya, "Combinatorial topologies for discrete planes", in LNCS 2886 : Discrete Geometry for Computer Imagery, Proceedings of 11th International Conference, DGCI2003, pp.144-153, Springer-Verlag, Heidelberg, 2003.

10. Y. Kenmochi, A. Imiya, "Combinatorial boundary tracking of a 3D lattice point set," submitted to Journal of Visual Communication and Image Representation.

11. D. G. Morgenthaler, A. Rosenfeld, "Surfaces in three-dimensional digital images," Information and Control, Vol. 51, pp. 227-247, 1981.

12. J. Stillwell, Classical Topology and Combinatorial Group Theory, Springer, New York, 1993.

13. G. M. Ziegler, Lectures on Polytopes, Springer, New York, 1995. 